\title{
CONFORMAL CIRCLES AND PARAMETRIZATIONS OF CURVES IN CONFORMAL MANIFOLDS
}

\author{
T. N. BAILEY AND M. G. EASTWOOD
}

(Communicated by Jonathan M. Rosenberg)

\begin{abstract}
We give a simple ODE for the conformal circles on a conformal manifold, which gives the curves together with a family of preferred parametrizations. These parametrizations endow each conformal circle with a projective structure. The equation splits into two pieces, one of which gives the conformal circles independent of any parameterization, and another which can be applied to any curve to generate explicitly the projective structure which it inherits from the ambient conformal structure [1].

We discuss briefly the use of conformal circles to give preferred coordinates and metrics in the neighborhood of a point, and sketch the relationship with twistor theory in the case of dimension four.
\end{abstract}

\section{INTRODUCTION}

On a Riemannian (or pseudo-Riemannian) manifold, one has a distinguished family of curves, the geodesics, with a unique geodesic issuing from any point with any specified velocity. On a conformal manifold there is also a distinguished family of curves, the conformal circles (also called conformal geodesics) [5], which are given by a third-order equation; and so there is a conformal circle through each point with a specified velocity and acceleration.

It is a result of Cartan [1] that any smooth curve in a conformal manifold (in the pseudo-Riemannian case it must have the property that its tangent vector is nowhere null) has a natural family of parametrizations with freedom PSL $(2, \mathbf{R})$. One usually constructs this family by means of the Cartan conformal connection.

In other treatments of conformal circles, they are either derived from the Cartan conformal connection or they are given directly by an ODE with no regard to the preferred parametrizations. In this note we give an ODE which gives the conformal circles directly with their preferred parametrizations. This equation may be split into two equations, one of which is parameterization invariant, and

Received by the editors August 1, 1988 and, in revised form, March 13, 1989 and May 11, 1989.

1980 Mathematics Subject Classification (1985 Revision). Primary 53A30; Secondary 58G30, $58 \mathrm{G} 35$.

This work was carried out with support from the Australian Research Council.

(C) 1990 American Mathematical Society $0002-9939 / 90 \$ 1.00+\$ .25$ per page 
the other can be applied to any curve to give its preferred parametrizations. This gives an elementary and direct proof of Cartan's result as mentioned above.

One consequence of the preferred parametrizations is that given a point together with the 1-jet at that point of a metric in the conformal class, one can use a construction like that for Riemann normal coordinates to construct a preferred coordinate system in a neighborhood of the point.

We also give a brief discussion of the conformally flat case and sketch the relationship with twistor theory.

\section{ASSUMPTIONS AND CONVENTIONS}

We will state a few assumptions here which we will make throughout in order to save repetition. The most important of these is that all considerations in this paper are local.

Our manifolds are smooth and come equipped with a Riemannian or pseudoRiemannian conformal structure-that is an equivalence class of metrics related by conformal transformations

$$
g_{a b} \mapsto \hat{g}_{a b}=\Omega^{2} g_{a b}
$$

By a curve we mean a smooth one-dimensional submanifold, with the restriction that in the pseudo-Riemannian case its tangent is nowhere null.

Our conventions for curvature are that given a nondegenerate metric $g_{a b}$, Riemannian or pseudo-Riemannian, on an $n$-dimensional manifold $M$, we write $\nabla_{a}$ for the unique torsion-free metric connection. The Riemann curvature is defined by $2 \nabla_{[a} \nabla_{b]} V^{d}=R_{a b c}{ }^{d} V^{c}$ (where the brackets denote antisymmetrization), and the Ricci tensor is defined by $R_{a c}=R_{a b c}{ }^{b}$.

Under change of metric according to equation (1), the associated metric connection transforms according to

$$
\hat{\nabla}_{a} V^{b}=\nabla_{a} V^{b}+\Upsilon_{a} V^{b}-V_{a} \Upsilon^{b}+\Upsilon_{c} V^{c} \delta_{a}^{b}
$$

where, throughout this paper,

$$
\Upsilon_{a}=\Omega^{-1} \nabla_{a} \Omega
$$

We define the curvature tensor

$$
P_{a b}=\frac{-1}{n-2}\left(R_{a b}-\frac{1}{2(n-1)} R g_{a b}\right)
$$

where $R=R_{a}^{a}$ is the scalar curvature, and $n$ is the dimension of the manifold. Under conformal transformation,

$$
\widehat{P}_{a b}=P_{a b}-\nabla_{a} \Upsilon_{b}+\Upsilon_{a} \Upsilon_{b}-\frac{1}{2} \Upsilon^{2} g_{a b}
$$

where, here and hereafter, we write $\Upsilon^{2}$ for $\Upsilon^{c} \Upsilon_{c}, U \cdot A$ for $g_{a b} U^{a} A^{b}$, etc. 
Our approach throughout is to work with the metric connection of some chosen metric in the conformal class and to check that our equations are independent of that choice as necessary.

\section{THE CONFORMAL CIRCLE EQUATION}

We will begin by defining conformal circles as solutions of an ODE. We will consider the equivalence of this to other definitions later. A derivation of this equation from the local twistor [4] form of the Cartan conformal connection will be given in a forthcoming paper.

Let $M$ be a conformal manifold, Riemannian or pseudo-Riemannian, and let $\gamma$ be a curve in $M$, parametrized by a variable $t$. Thus, $\gamma$ is a smooth one-dimensional submanifold equipped with a smooth function $t: \gamma \rightarrow \mathbf{R}$. Let $U^{a}$ be the velocity vector of the curve, so that $U^{a} \nabla_{a} t=1$, and let $A^{b}$ be the acceleration vector $A^{b}=U^{a} \nabla_{a} U^{b}$. We note that $A^{b}$ is not conformally invariant, but from equation (2),

$$
\hat{A}^{b}=A^{b}-U^{2} \Upsilon^{b}+2\left(U^{c} \Upsilon_{c}\right) U^{b} .
$$

Definition 3.1. A curve $\gamma$ is a conformal circle if it satisfies

$$
U^{a} \nabla_{a} A^{b}=\frac{3 U \cdot A}{U^{2}} A^{b}-\frac{3 A^{2}}{2 U^{2}} U^{b}+U^{2} U^{a} P_{a}^{b}-2 P_{c d} U^{c} U^{d} U^{b} .
$$

We recall here our convention that in case $M$ is pseudo-Riemannian we consider only curves with non-null tangent. It can be verified directly using (2), (3), (4) that the equation is conformally invariant.

In the following lemma the conformal circle equation is split into two separate conformally invariant equations. The proof is trivial.

Lemma 3.2. The conformal circle equation is equivalent to the pair of equations

$$
\begin{gathered}
U_{b} U^{a} \nabla_{a} A^{b}=\frac{3(U \cdot A)^{2}}{U^{2}}-\frac{3 A^{2}}{2}-U^{2} U^{a} U^{b} P_{a b} \\
U^{c} \nabla_{c}\left(A_{[b}\right) U_{a]}=\frac{3(U \cdot A)}{U^{2}} U_{[a} A_{b]}+U^{2} U^{c} P_{c[b} U_{a]} .
\end{gathered}
$$

The following proposition provides an alternative, somewhat more geometric, definition of conformal circles. ${ }^{*}$

Proposition 3.3. A curve is a conformal circle if and only if there exists a metric in the conformal class such that the curve is an affinely parametrized geodesic and $U^{a} P_{a b}=0$.

Proof. If $\gamma$ is such a curve, then we have $A^{b}=0$ in the metric assumed in the proposition and the conformal circle equation is clearly obeyed. Conversely,

* The origin of this definition is not clear, it was known to R. Penrose some years ago, but he believes he found it in the literature. See also [2]. 
suppose we are given a curve satisfying the conformal circle equation. We can always choose a metric locally so that the curve is an affinely parametrized geodesic. Reference to the conformal circle equation in the form given by Lemma 3.2 then gives us that $U^{a} U^{b} P_{a b}=0=U^{c} P_{c[b} U_{a]}$. This implies $U^{a} P_{a b}=$ 0 in such a metric, which completes the proof. The geometric relationship of this definition to Cartan's conformal connection will be made clear in a forthcoming paper by the authors.

One might ask, given the comparative rarity of conformally invariant objects, whether the conformal circle equation is the only third-order equation for a curve naturally definable on a conformal manifold. The answer is that there is at least the freedom to use the conformally invariant vector

$$
K^{e}=g^{e c} U^{a} U^{b}\left(C_{b c a}{ }^{d} A_{d}-2 U^{2} \nabla_{[c} P_{a] b}\right)
$$

where $C_{a b c}{ }^{d}$ is the Weyl tensor, in the equation whenever $M$ is not conformally flat.

Further, one might ask whether the conformal circle equation (5) arises from a variational principle. Since it is third order, it cannot directly arise from a nondegenerate Lagrangian. However, there is a natural Lagrangian

$$
L=\frac{A^{2}}{U^{2}}-\frac{2(U \cdot A)^{2}}{U^{4}}+2 P_{a b} U^{a} U^{b}
$$

which transforms under conformal rescaling according to

$$
\widehat{L}=L-2 U^{a} \nabla_{a}(U \cdot \Upsilon)
$$

One can thus ask for parametrized curves with specified ends, and given velocities at those ends, which make the integral of $L$ along the curve extremal. This leads to a forth-order conformally invariant ODE, but conformal circles obey this if and only if the invariant vector $K^{e}$ vanishes, as it does in the conformally flat case.

\section{REPARAMETRIZATIONS}

A reparametrization of $\gamma$ with a new variable $s=g(t)$ is accompanied by the replacement $U^{a} \mapsto \widetilde{U}^{a}=\left(g^{\prime}\right)^{-1} U^{a}$. If one then calculates the resulting change in $A^{b}$, etc. it is easy to see that equation (7) is invariant.

If we now set

$$
f=U_{b} U^{a} \nabla_{a} A^{b}-\frac{3(U \cdot A)^{2}}{U^{2}}+\frac{3 A^{2}}{2}+U^{2} U^{a} U^{b} P_{a b}
$$

so that $f=0$ is equation (6), we discover that under reparametrization

$$
f \mapsto \tilde{f}=\left(g^{\prime}\right)^{-4} f+U^{2}\left[\left(g^{\prime}\right)^{-5} g^{\prime \prime \prime}-\frac{3}{2}\left(g^{\prime}\right)^{-6}\left(g^{\prime \prime}\right)^{2}\right] .
$$

Since $\tilde{f}=0$ is an ordinary differential equation, we can always locally reparametrize any curve so that it satisfies equation (6); the freedom in so doing is the 
solutions of

$$
\left(g^{\prime}\right)^{-5} g^{\prime \prime \prime}-\frac{3}{2}\left(g^{\prime}\right)^{-6}\left(g^{\prime \prime}\right)^{2}=0 .
$$

This is the Schwarzian differential equation, which has as its solutions

$$
g(t)=\frac{a t+b}{c t+d} \quad \text { where }\left(\begin{array}{ll}
a & b \\
c & d
\end{array}\right) \in \operatorname{SL}(2, \mathbf{R}) .
$$

We have thus proved:

Proposition 4.1. Any curve in a conformal manifold with a tangent vector of nowhere zero length has (at least locally) a conformally invariant preferred family of parametrizations, with the freedom of $\operatorname{PSL}(2, \mathbf{R})$, the projective group of the line.

This gives an elementary and explicit proof of Cartan's observation [1] that a curve in a conformal manifold inherits a natural projective structure. We shall call such preferred parametrizations projective.

To return to conformal circles, we have proved the following:

Proposition 4.2. A curve $\gamma$ can be reparametrized to obey the conformal circle equation (5) if and only if it obeys equation (7).

The usual equation for conformal circles [5] is (translated into our conventions),

$$
U^{c} \nabla_{c} A^{b}=-A^{2} U^{b}+U^{c} P_{c}^{b}-U^{c} U^{d} P_{c d} U^{b} .
$$

It is easy to check that this is equivalent to equation (7) together with the requirement that the curve is parametrized by arc length in the metric in which one is working. This does not seem to be the most geometric choice of parametrization.

Since the defining equation is third order, there is a unique projectively parametrized conformal circle through a given point with given initial velocity and acceleration. Thus, the space of projectively parametrized conformal circles is $3 n$ dimensional, and the space of unparametrized conformal circles is $3 n-3$ dimensional.

\section{CONFORMAL COORDINATES}

As we pointed out above, the acceleration of a conformal circle is not conformally invariant; it is well defined, however, if one makes a choice of metric in the conformal class. In fact, all one needs to define the acceleration at a point is a 1 -jet of a metric at that point.

Given any point $p \in M$ and a choice of a 1-jet of a metric in the conformal class, we can single out a special set of projectively parametrized conformal circles through $p$, namely those with unit length velocity and zero acceleration at $p$. We can then use these in exactly the same way as we do the geodesics in the construction of Riemann normal coordinates to construct a preferred coordinate system in a neighborhood of $p$. We will call them conformal circle 
coordinates. These coordinates have been used in general relativity theory by Friedrich and Schmidt [2].

Preferred coordinate systems depending only on a metric 1 -jet at a point have also been constructed by Graham [3]; the relationship between his construction and ours remains obscure, but the conformal circle coordinates do have the advantage of existing in a neighborhood of the point, rather than just as a power series at the point.

One might also consider using the special metric in the conformal class, defined so that $g_{a b} U^{a} U^{b}=1$, thus obtaining a preferred metric in a neighborhood of $p$ from knowing only a metric 1 -jet at $p$, but such metrics will not generally be smooth at $p$.

\section{Conformally flat SPACE}

The $n$-sphere with the usual round metric is conformally flat, and it is easy to see that the conformal circles are just the 'round' circles (obtained by cutting the sphere embedded in $\mathbf{R}^{n+1}$ with any plane). Their projective parametrizations are obtained by projecting from any point on the circle to the tangent line of any other point. We can complexify this picture, to obtain the conformal circles in the complexified $n$-sphere; these will be holomorphic curves satisfying the complex version of equation (5).

In the special case $n=4$, we can use the twistor construction (see e.g. [4]) to investigate the geometry further. Given a four-dimensional complex vector space $T$, the Grassmannian $\operatorname{Gr}(2, T)$ of all two-dimensional subspaces of $T$, has a natural conformal structure given by nontrivial intersection in $T$, which is precisely that of the complexified 4-sphere $\mathrm{CS}^{4}$.

Now let $P$ be the projective space of $T$, so that $C S^{4}$ can be identified with the space of projective lines in $P$. Any ruled surface in $P$ gives a one-parameter family of lines in $P$, and hence a curve in $C S^{4}$. A particular family of ruled surfaces is the nine-dimensional space of nondegenerate quadrics, ruled by their generators; and these give precisely the (unparametrized) conformal circles in $\mathrm{C} S^{4}$.

There is a general relationship between twistor theory and conformal circles, which will be explained as part of a forthcoming paper of the authors on the general theory of local twistors.

\section{ACKNOWLEDGMENT}

Professor T. N. Bailey would like to thank the University of Adelaide for hospitality during this time.

\section{REFERENCES}

1. É. Cartan, Les espaces à connexion conforme, Oeuvres Complètes III.1, Gauthiers-Villars, Paris, 1955, pp. 747-797.

2. H. Friedrich and B. G. Schmidt, Conformal geodesics in general relativity, Proc. Roy. Soc. London Ser. A 414 (1987), 171-195. 
3. R. Graham, A conformal normal form, (to appear).

4. R. Penrose and W. Rindler, Spinors and space-time Vol. 2, Cambridge University Press, 1986.

5. K. Yano, The theory of the Lie derivative and its applications, North Holland, Amsterdam, 1955.

Department of Mathematics, University of Edinburgh, Edinburgh, United Kingdom Department of Mathematics, University of Adelaide, Adelaide, South Australia 